\title{
Characterization of AlNi formed by a self-sustaining reaction during mechanical alloying
}

\author{
Michael Atzmon \\ Department of Nuclear Engineering, The University of Michigan, Ann Arbor, MI 48109-2104 (U.S.A.)
}

\begin{abstract}
We report on electron-microscopic studies of AlNi powder formed by an explosive reaction during mechanical alloying. In situ thermal analysis enabled us to terminate the milling process immediately following the reaction, so that further mechanical damage to the reaction product was minimized. The surfaces of the resulting particles are smooth on a scale of tens of nanometers. We suggest that explosive reactions occur in individual grains during mechanical alloying even for seemingly gradual reactions, and that amorphous phase formation could involve such mechanism.
\end{abstract}

\section{Introduction}

High-energy ball milling has been traditionally used to mix powder particles and to control the particle size. In recent years it has been discovered that the same technique can be used to achieve atomic-scale alloying of elemental powders [1-3]. Such mechanical alloying (MA) can result in the formation of equilibrium $[1,3]$ or metastable [2] alloys, the latter being amorphous or crystalline. It has generally been assumed that the MA process occurs by solid-state diffusion. Estimates of the peak temperatures during ball milling have led previous authors to the conclusion that the occurrence of melting is unlikely [4]. We have also shown [5] that elemental composites can be reacted and amorphized by rolling at nearambient temperatures. It has been suggested that high densities of plane and line defects, as well as supersaturation of point defects, created during mechanical deformation, can assist solid-state interdiffusion. Because of the obvious experimental limitations, characterization techniques applied to ball-milled samples originally were $e x$ situ, so that the alloying process was characterized only by its final product.

Recently, the author has reported [6] in situ thermal characterization of the reaction of an equiatomic mixture of aluminum and nickel powders to form crystalline intermetallic compounds. Under proper conditions, if the milling process was interrupted and subsequently resumed, a complete reaction took place within 2-3 s. This was evidenced by a sudden increase of the vial temperature, and a complete transformation into AlNi was confirmed by X-ray diffraction. The measured value of the temperature rise was in agreement with data on the enthalpy of formation of AlNi. The results, coupled with thermochemical estimates, strongly suggest the possibility of melting during reaction. More recently, the author [7] has also shown that the phase formation kinetics are very sensitive to the sample temperature and to the atmosphere in the vial.

A significant result of the in situ thermal characterization technique was the ability to stop the milling process as soon as a reaction took place, thus minimizing the deformation of the reaction product. This allows us to study the reaction product and learn more about the details of its formation process. In this paper we report on scanning electron microscopy (SEM) and transmission electron microscopy (TEM) studies of the product of such self-sustained reaction. In contrast to unreacted particles, the reacted AINi has surfaces which are smooth on a scale of tens of nanometers. We further suggest that AINi is always formed by self-sustained reaction, including the case of seemingly gradual transformations.

\section{Experimental details}

A SPEX 8000 vibratory ball mill was used for sample preparation (for details see ref. 6). 
Samples were obtained by stopping the ball milling process within less than a second of the observation of the exothermic peak. Electron microscopy was performed with a Hitachi S 520 SEM and JEOL 2000FX STEM, both equipped with X-ray fluorescence analysis. The following process was used to prepare TEM specimens: the sample powder was embedded into a tin foil by cold-rolling. Subsequently, a $0.25 \mathrm{~mm}$ thick nickel layer was electrodeposited onto the powder side of the tin foil and the tin foil was mechanically removed. The powder, partly embedded in the nickel foil, was then coated with a nickel layer of equal thickness on its other side. Discs of $3 \mathrm{~mm}$ were cut from the resulting nickel foil, and these were mechanically thinned and subsequently ion-milled to produce electrontransparent regions.

\section{Results and discussion}

The surface of particles before and after the exothermic reaction were investigated by SEM. As shown previously [6], ball milling of elemental $\mathrm{Al}_{50} \mathrm{Ni}_{50}$ powder mixture for $60-120$ min results in a lamellar structure consisting of unreacted elemental aluminum and nickel. Figure 1 shows a secondary electron image of the surface of such a particle, revealing its typical surface roughness. Figure 2(a) shows secondary electron images of a particle obtained for the same sample when stopping the milling immediately after observing the exothermic reaction. The particle is approximately $1 \mathrm{~mm}$ large and appears to be an aggregate of many smaller particles. Figures 2(b), 2(c), and 2(d) show magnified images of typical regions in the same particle. With the exception of some 1000-2000 $\AA$ large particles, the surface appears smooth at a resolution of $500 \AA$. Such smooth surface suggests that this part of the particle did not fracture or undergo mechanical deformation after the temperature spike. The smoothness is a result of the high-temperature transient during milling, either by flow in the liquid state, or by rapid diffusion in the solid state. The small particles protruding from the surface have not been identified, and are suggested to be either smaller powder particles attached to the surface, or oxide particles which were not dissolved in the matrix during the formation of AlNi. Figure 2(d) shows surface regions divided by smooth lines which appear to be grooves at grain boundaries, with grain sizes of approximately $2 \mu \mathrm{m}$.

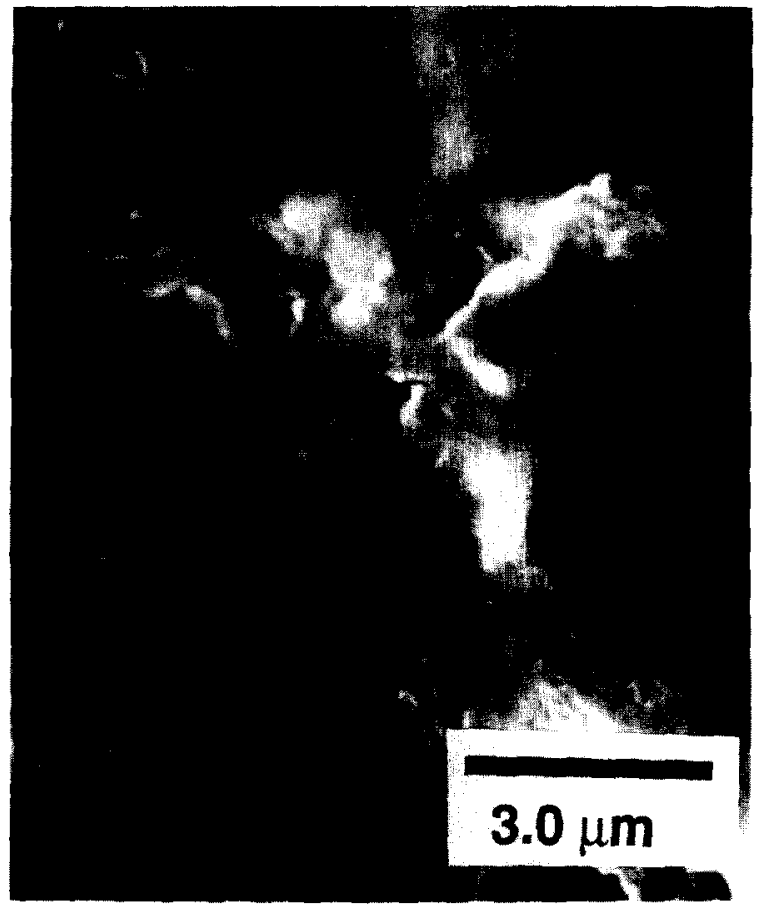

Fig. 1. SEM micrograph of an $\mathrm{Al}_{50} \mathrm{Ni}_{\xi(1)}$ powder particle after milling for $60 \mathrm{~min}$.

In the TEM analysis, the AlNi phase was distinguished from the nickel, in which it was embedded, using X-ray fluorescence analysis. Grain diameers of several microns were observed. Figure 3 shows a TEM micrograph obtained with the beam in the $[100]$ direction. The visible dislocation lines form an approximate grid. The electron diffraction pattern for this region corresponds to the symmetry of $\mathrm{NiAl}$ (CsCl type) and contains superlattice spots.

We finally comment on the mechanism of AINi formation. We have previously shown [7] that, depending on the milling temperature and atmosphere, elemental $\mathrm{Al}_{50} \mathrm{Ni}_{50}$ powder mixture reacts to form AlNi either explosively or gradually. In isothermal diffusion couple experiments, however, $\mathrm{Al}_{3} \mathrm{Ni}$ is the first phase to form $[7,8]$. We suggest, therefore, that the apparent gradual formation of AlNi occurs by self-sustained reactions in individual particles. Before reaction, each particle, consisting of many elemental lamellae, has the same average composition as the entire sample. Therefore, when a particle reaches equilibrium during a temperature spike, AINi forms and $\mathrm{Al}_{3} \mathrm{Ni}$ formation is bypassed. It is possible that an undetected, self-sustained reaction is attained in other alloy systems with large, nega- 

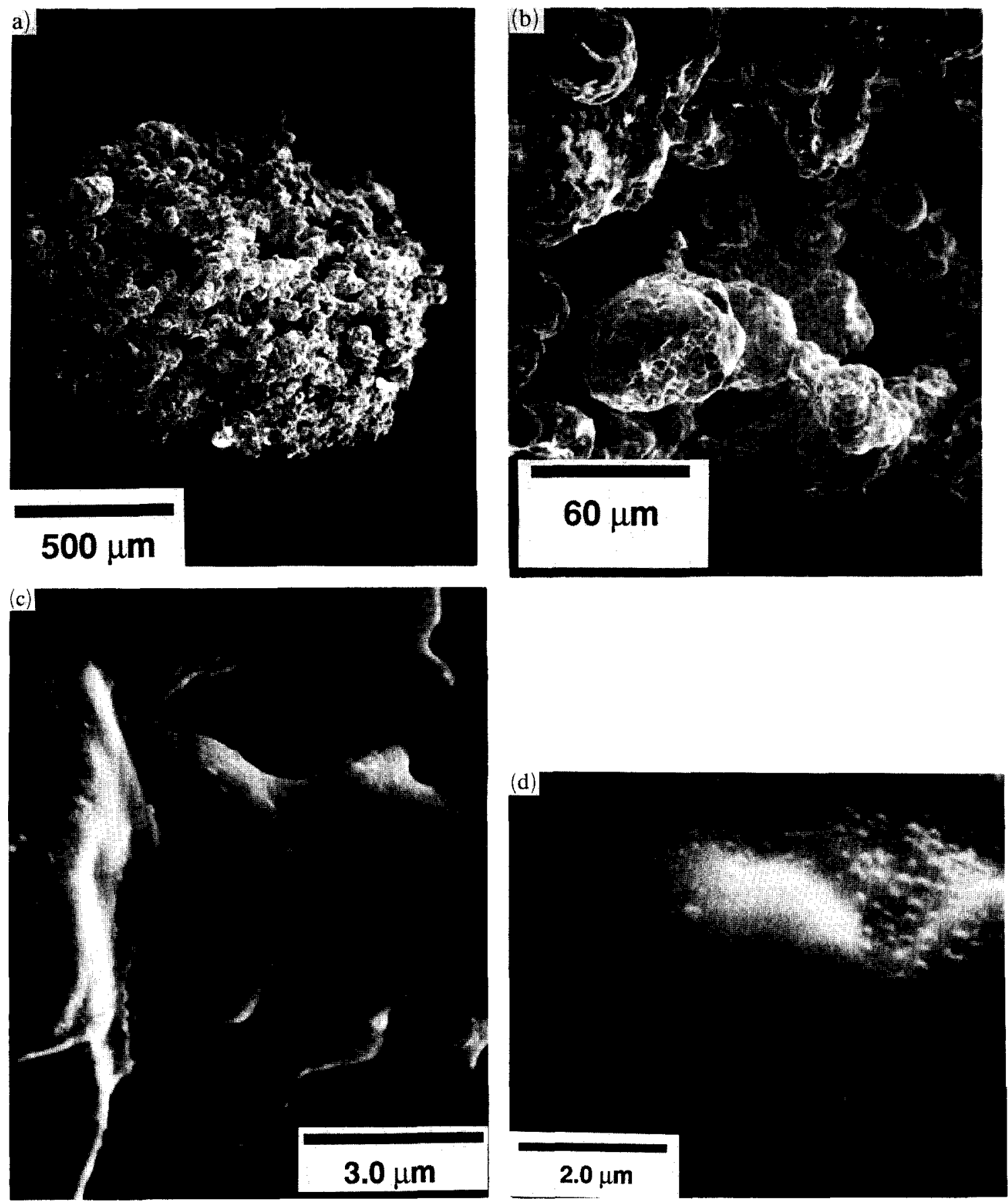

Fig. 2. SEM micrographs of an $\mathrm{Al}_{50} \mathrm{Ni}_{50}$ powder particle immediately after an exothermic reaction, taken with different magnifications.

tive heats of reaction. We suggest the possibility that amorphous alloys form by such melting followed by rapid solidification.

\section{Conclusion}

We have presented results of electron-microscopic investigations of $\mathrm{AlNi}$ powder formed by explosive reaction during mechanical alloying. The smooth surfaces and large grain sizes suggest that the sample particle did not undergo significant mechanical damage after its formation. The possibility of undetectable explosive reactions is suggested as a possible alloying mechanism in other systems. 


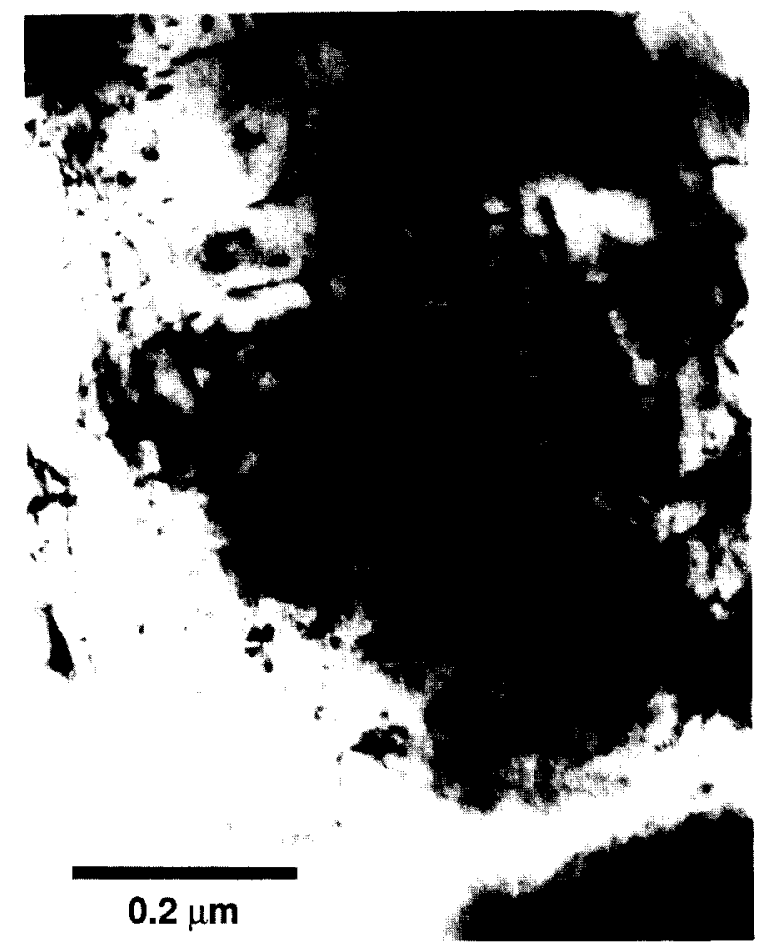

Fig. 3. TEM micrograph of the same sample as in Fig. 2.

\section{Acknowledgments}

The author acknowledges experimental assistance by S. Hise and T. Al-Nahlawi. This work was supported in part by the National Science Foundation, grant No. DMR-8820285.

\section{References}

1 J. S. Benjamin and R. D. Schelleng, Metall. Trans. A, 12 (1981) 1827

2 C. C. Koch, O. B. Cavin, C. G. McKamey and J. O. Scarborough, Appl. Phys. Lett., 43(1983) 1017.

3 E. Ivanov, T. Grigorieva, G. Golubkova, V. Boldyrev, A. B. Fasman, S. D. Mikhailenko and O. T. Kalinina, Mater. Lett., 7(1988) 51.

4 R. B. Schwarz, R. R. Petrich and C. K. Saw, J. Non-Crystalline Solids, 76(1985) 281.

5 M. Atzmon, K. M. Unruh and W. L. Johnson, J. Appl. Phys., 56(1985) 3865.

6 M. Atzmon, Phys. Rev. Lett., $64(1990) 487$.

7 M. Atzmon, in A. H. Clauer and J. J. deBarbadillo (eds.), Solid State Powder Processing. The Minerals, Metals and Materials Society, Warrendale, 1990, p. 173.

8 E. G. Colgan. M. Nastasi and J. W. Mayer, J. Appl. Phys, 58 (1985) 4125 